\title{
Endometrial resection following levonorgestrel intrauterine system treatment for menorrhagia
}

\author{
Pentti K. Heinonen • Riikka Helin
}

Received: 12 November 2008 / Accepted: 18 February 2009 / Published online: 7 March 2009

(C) Springer-Verlag 2009

\begin{abstract}
The objective of this study was to evaluate longterm outcomes and hysterectomy rates after hysteroscopic endometrial resection among women following use of a levonorgestrel-releasing intrauterine system (LNG-IUS) for menorrhagia and among women never using this. Forty-five women who had used LNG-IUS for treatment of menorrhagia underwent endometrial resection. Each of them was matched with a patient who had never used LNG-IUS and who had been treated by endometrial resection for menorrhagia (control group). These two groups were compared for operative outcomes, additional procedures, outcome of menstrual bleeding, and for subsequent hysterectomy, which was the endpoint of this study. During the mean follow-up period of 5.8 years, 19 (44\%) women undergoing endometrial resection following LNG-IUS and $14(31 \%)$ with endometrial resection and without use of LNG-IUS underwent at least one gynecological procedure. Hysterectomy was performed in $14(33 \%)$ women who had used LNG-IUS and in seven (16\%) in the control group $(p=0.05)$. The main indications for hysterectomy were pain and enlarged uterus; leiomyomas and/or adenomyosis were found in specimens of the uterus extirpated, except for two cases with hematometra in both groups. Patients not hysterectomized reported amenorrhea or slight bleeding, and this response persisted for years after the treatment. In conclusion, two of three women avoided hysterectomy
\end{abstract}

P. K. Heinonen $(\bowtie) \cdot$ R. Helin

Medical School, University of Tampere,

Tampere 33014, Finland

e-mail: pentti.heinonen@uta.fi

P. K. Heinonen

Department of Obstetrics and Gynecology,

Tampere University Hospital,

Tampere 33521, Finland when endometrial resection followed LNG-IUS although hysterectomy rate was higher than those who had never used the device. Hysteroscopic surgery may be considered as an alternative to hysterectomy after discontinuation of LNG-IUS for menorrhagia.

Keywords Endometrial resection · Hysterectomy · Hysteroscopy · Levonorgestrel-releasing intrauterine system $\cdot$ Menorrhagia

\section{Introduction}

The levonorgestrel-releasing intrauterine system (LNGIUS) has been used for contraception, as a treatment for heavy menstrual bleeding and as the progestogen component in hormone replacement therapy. It induces atrophy of the endometrial epithelium and thus reduces menstrual bleeding in menorrhagic women. LNG-IUS is one of the most acceptable drug treatment options for women suffering from menorrhagia at reproductive age $[1,2]$.

Hysteroscopic surgery, including endometrial resection or ablation, myomectomy, and polypectomy, is a valid surgical alternative to hysterectomy in the treatment of menorrhagia [3]. Endometrial resection is not suitable for women who wish to preserve their fertility. Both LNG-IUS and endometrial resection effectively reduced menstrual bleeding in a randomized study with 36 months follow-up [4].

LNG-IUS has been associated with a high discontinuation rate, $50 \%$ of women having had the system removed within 4 to 5 years from insertion $[5,6]$. A high hysterectomy rate was reported among women initially treated by LNG-IUS in a 5-year trial [6]. Hysteroscopic surgery instead of hysterectomy has not been studied as a surgical option among women from whom LNG-IUS failed as treatment of menorrhagia. In 
this paper, we report our experience of endometrial resection in women after unsuccessful use of LNG-IUS.

\section{Materials and methods}

The study group (LNG-IUS group) comprised 45 premenopausal women who had used LNG-IUS (Mirena ${ }^{\circledR}$, Schering Co, Turku, Finland) for menorrhagia prior to hysteroscopic endometrial resection in Tampere University Hospital, Tampere, Finland. Each of these women was matched for age, parity, and nearest date of operation with a patient who had never used LNG-IUS and was treated by endometrial resection in the same period (control group). Table 1 shows patient characteristics in both groups.

The reason for poor response or decision to remove LNG-IUS was based on adverse effects in 17 (38\%) cases, no response in menstrual bleeding in 14 (31\%), and expulsion of intrauterine device in 11 (24\%) cases. Two women experienced pelvic pain, and one was diagnosed breast cancer and the device was removed. The mean duration of use of LNG-IUS was 16 months (range 154 months). Twenty-nine women had removed or expelled the device on average 7.5 months (range 1-47 months) prior to hysteroscopic surgery.

All patients had primarily suffered from menorrhagia and they were offered hysteroscopic surgery instead of hysterectomy. Cervical smear and endometrial biopsy were taken preoperatively. Women with a uterus larger than the size of a 12-week pregnancy, endometrial hyperplasia, untreated adnexal disease, or acute pelvic inflammatory disease and those desiring future pregnancy were excluded from hysteroscopic surgery.

Sixteen out of 45 women had the LNG-IUS in place and it was removed prior to hysteroscopic surgery. Fifteen out of 21 women who had removed or expelled the device in the study group and 21 women in the control group had received $600 \mathrm{mg}$ danazol daily for 4 weeks preoperatively to suppress the endometrium (Table 1). Fourteen patients in the study group and 24 in the control group had no suppression of the endometrium with hormonal therapy and their hysteroscopic surgery was performed on days 4-9 of the menstrual cycle.

The procedure was carried out under spinal anesthesia in 40 cases $(44 \%)$. The uterine cavity was distended with $2.2 \%$ glycine (Baxter Health Care Ltd., Thetford, UK) and the endometrium resected using a 26-Fr unipolar resectoscope as previously described [7]. Twenty-three women (25.6\%) underwent concomitant hysteroscopic myomectomy and 23 (25.6\%) laparoscopic tubal ligation. Antibiotic prophylaxis was not routinely used.

Hospital records of the patients were examined for details of preoperative history, operative procedures, further surgical treatment, and documented follow-up. Patients who had not undergone hysterectomy during the follow-up period were sent a postal questionnaire to assess long-term effects. Questions concerned the need for further treatment, hormonal treatment, bleeding patterns, and success of treatment. Respondents were asked whether they had amenorrhea, slight menstruation for 1-3 days, no improvement in menstrual flow, or increased menstrual flow.

Follow-up data were received from 88 patients. One patient had died and one was lost to follow-up both in the LNG-IUS group. The mean (standard deviation, SD) follow-up period was 5.4 (2.9) years in the LNG-IUS group and 6.2 (2.0) years in the control group.

Statistical analysis was made using the SPSS for Windows, version SPSS 11.5 (SPSS Inc., Chicago, IL, USA). Subsequent hysterectomy was the endpoint and its incidence was calculated by survival analysis (Kaplan-Meier) in both groups and the curves compared by log-rank test. For statistical analysis, unpaired Student's test for continuous data and Fisher's exact test for nominal or ordinal data were used. The level of significance was set at $p<0.05$.

\section{Results}

Women in the control group had a history of tubal ligation for sterilization more often $(p=0.03)$ than those in the LNG-IUS group. Patients in the study group had received treatment (LNG-IUS in situ or danazol) to reduce endome-
Table 1 Baseline demographic and clinical characteristics of women with endometrial resection with or without prior use of levonorgestrel intrauterine system

$* p=0.05 ; * * p=0.03$

\begin{tabular}{lll}
\hline Characteristics & LNG-IUS $(n=45)$ & Control $(n=45)$ \\
\hline Age, year, mean (SD) & $40.9(3.9)$ & $41.3(3.8)$ \\
Body mass index, $\mathrm{kg} / \mathrm{m}^{2}$, mean (SD) & $27.1(5.2)$ & $25.3(3.4)$ \\
Parity, median (range) & $2(0-5)$ & $2(0-4)$ \\
Cesarean section $n(\%)$ & $3(6.7)$ & $3(6.7)$ \\
Sterilization $n(\%)$ & $16(35.6)$ & $27(60.0)^{* *}$ \\
Endometrial thinning $n(\%)$ & $31(68.9)^{*}$ & $21(46.7)$ \\
LNG-IUS in situ & 16 & 0 \\
Danazol & 15 & 21 \\
\hline
\end{tabular}


trium thickness more frequently $(p=0.05)$ than controls. Otherwise, the characteristics of patients were similar in both groups (Table 1).

Table 2 shows operative details and operative complications in both groups. Laparoscopic tubal ligation subsequent to endometrial resection was performed in $18(40 \%)$ cases using LNG-IUS and in five (11\%) women in the control group $(p=0.004)$. Severe complications did not occur in both groups, but six patients in the study group and two in the control group had minor complication $(p=0.27)$. Three (6.7\%) women in the study group had postoperative infection and they recovered completely after oral antibiotic treatment. Of 40 women receiving spinal anesthesia, five $(12.5 \%)$ suffered from postspinal headache, which was successfully treated with a blood patch (Table 2).

At least one abnormal finding in the tissue specimens collected after resection was reported in $19(42 \%)$ cases using LNG-IUS and in $16(36 \%)$ controls. Concomitant hysteroscopic resection of fibroids was performed in 13 (29\%) and polypectomy in three $(7 \%)$ cases with use of LNG-IUS and in ten $(22 \%)$ and in five $(11 \%)$ cases in the control group, respectively. Adenomyosis was detected in two (4\%) specimens from the study group and in three (7\%) in the control group. Endometrial hyperplasia was found in one $(2 \%)$ specimen in both groups.

During the follow-up period, 19 (44\%) out of 43 women with LNG-IUS and 14 (31\%) out of 45 women without LNG-IUS underwent at least one gynecological procedure $(p=0.38)$. Endometrial reablation was performed in three cases $(7 \%)$ in the study group and in four (9\%) in control group because of hematometra (Table 3).

Hysterectomy was performed in 14 (33\%) cases among LNG-IUS users and in seven (16\%) in the control group. Survival analysis showed in both groups a relationship between the probability of not undergoing subsequent hysterectomy and the time since the endometrial resection procedure (Fig. 1). The difference between the groups was statistically significant (log-rank test, $p=0.05$ ).

The main indications for subsequent hysterectomy were pain and enlarged uterus. Twelve women in the LNG-IUS group and five controls were found leiomyomas and/or adenomyosis in specimens of the uterus extirpated. Two patients in the study group and two in the control group had hematometra. One patient in the LNG-IUS group had endometrial hyperplasia, but no cases of uterine malignancy were found.

Long-term follow-up data were available for 67 women who did not require hysterectomy. Eighteen women in the LNG-IUS group and 24 in the control group were premenopausal at the end of the follow-up period. Six women reported amenorrhea and 12 slight menstrual bleeding, four and 20 in the control group, respectively.

Eleven women in the LNG-IUS group and 14 of the controls had reached menopause during the follow-up period. Their mean (SD) age was 52.4 (2.0) years and they had menopausal symptoms. Six out of 11 women in the study group had used a cyclical combined estrogen and progesterone regimen: Two of them had amenorrhea and four slight bleeding, and eight out of 14 in the control group had five and three, respectively. Other postmenopausal women (five LNG-IUS and six controls) had amenorrhea. They had not received hormonal replacement therapy or they had used a continuous combined regimen. Uterine malignancy had not been diagnosed in any case.

\section{Discussion}

Long-term outcome of hysteroscopic surgery after unsuccessful use of LNG-IUS has not been previously reported. Hysterectomy has been the subsequent treatment if use of a progestin-releasing intrauterine device for menorrhagia has failed. One study has shown that $43 \%$ out of 119 women with LNG-IUS for menorrhagia had undergone hysterectomy during 5 years of follow-up [6]. The present study showed endometrial resection to be a potential alternative among these patients. Sixty-seven percent of women with discontinuation of LNG-IUS avoided hysterectomy when hysteroscopic surgery was performed for treatment of menorrhagia.
Table 2 Operative details and perioperative and postoperative complications among women used a levonorgestrel intrauterine system prior to endometrial resection and those who never used this

$* p=0.004$

\begin{tabular}{lll}
\hline & LNG-IUS $(n=45)$ & Control $(n=45)$ \\
\hline Length of uterine cavity, cm (SD) & $8.8(0.8)$ & $8.8(0.8)$ \\
Duration of operation, min (SD) & $32(14)$ & $28(12)$ \\
Glycine used, L (SD) & $5.5(2.4)$ & $4.8(1.8)$ \\
Glycine deficit >1 L (\%) & $1(2)$ & 0 \\
Myomectomy n (\%) & $13(19)$ & $10(22)$ \\
Tubal ligation n (\%) & $18(40)^{*}$ & $5(11)$ \\
Complication n (\%) & $6(13)$ & $2(4)$ \\
Infection & 3 & 0 \\
Postspinal headache & 3 & 2 \\
\hline
\end{tabular}




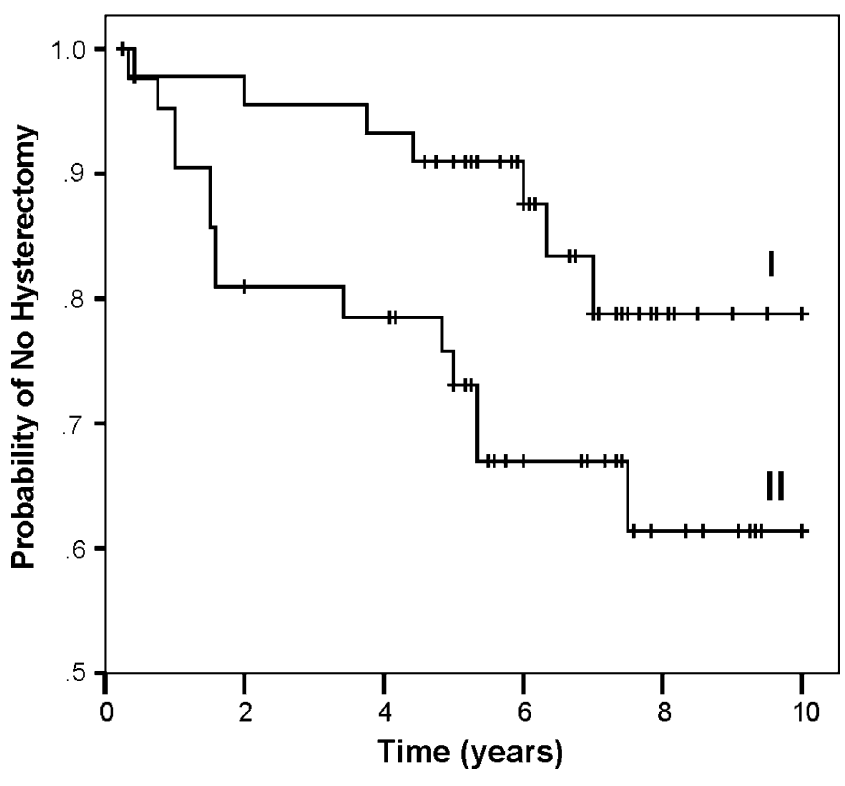

Fig. 1 Probability of not having hysterectomy in patients with endometrial resection without prior use of a levonorgestrel-releasing intrauterine system $(I)$ and in patients with endometrial resection following use of a levonorgestrel-releasing intrauterine system for menorrhagia (II). Kaplan-Meier curves are illustrated (log-rank test, $p=0.05$ )

Subsequent hysterectomy was selected as the endpoint in the study as hysterectomy rates may properly reflect the long-term results of a hysteroscopic procedure in women suffering from menorrhagia. After endometrial resection, hysterectomy rates of $15-24 \%$ have been reported with a follow-up period of 4 years or more [8-11]. In the present study, women who had used LNG-IUS prior to endometrial resection had higher hysterectomy rate $(33 \%)$ than those who had never used the device. Nineteen $(42 \%)$ women in the LNG-IUS group and 16 (36\%) controls had at least one abnormal finding in endometrial specimens, mostly submucous fibroids. Also indications for hysterectomy and pathologic findings in the uterine specimens after hysterectomy were similar in both groups, although the small number of cases limits definitive conclusions.

Endometrial resection offers an alternative to hysterectomy as a surgical treatment for heavy menstrual bleeding [12]. Endometrial resection has also been compared to
LNG-IUS in the treatment of menorrhagia. Rauramo and associates reported that both treatment modes effectively reduced menstrual bleeding in an open randomized 3-year trial [4].

Tubal ligation for sterilization prior to endometrial resection was more common among women in the control group, but among those who had used LNG-IUS, this procedure was more common during hysteroscopic surgery. LNG-IUS can also be used for contraception. It is also suitable for women who wish to preserve their fertility. Endometrial resection is possible only for women who do not wish to conceive and it is a real alternative to hysterectomy. Contraception is therefore imperative for those who have undergone endometrial resection for menorrhagia.

Pretreatment with medication reducing endometrial thickness may facilitate hysteroscopic surgery, improving visibility by limiting blood loss [13]. The progestin-releasing intrauterine system causes atrophy of the endometrium and it can be used as a preoperative hormonal treatment. LNG-IUS should be left in until the time of surgery as it ensures a thin endometrium which in turn facilitates the procedure.

We used the endometrial resection technique. Submucous myomas and polyps were common in both groups and it is possible to treat these by resection. Nowadays secondand third-generation ablation techniques have been introduced and found equivalent to the classical hysteroscopic procedures [14].

Most of the women in both groups avoided hysterectomy during the long follow-up period. Most of them had reached menopause. Postmenopausal women using a cyclic combined estrogen progestogen regimen may experience bleeding. In the present study, all subjects reported slight bleeding or amenorrhea. These findings indicate that the long-term impact is permanent and endometrial resection after use of LNG-IUS does not simply postpone hysterectomy and is also an alternative approach after failed use of LNG-IUS.

In conclusion, most women (67\%) with discontinuation of LNG-IUS avoided hysterectomy when endometrial resection was performed for treatment of menorrhagia, although those who had used LNG-IUS prior endometrial resection had higher hysterectomy rate than those who had never used the device.
Table 3 Further surgery after endometrial resection in women using LNG-IUS and in those not using it

\begin{tabular}{lll}
\hline Treatment & LNG-IUS $(n=43)$ & Control $(n=45)$ \\
\hline Any further treatment & $19(44 \%)$ & $14(31 \%)$ \\
Hysterectomy & 14 & 7 \\
Endometrial reablation & 3 & 4 \\
Hysteroscopy, curettage & 2 & 2 \\
Cervical dilatation and drainage & 2 & 2 \\
\hline
\end{tabular}




\section{References}

1. Oehler MK, Rees MCP (2003) Menorrhagia: an update. Acta Obstet Gynecol Scand 82:405-422

2. Marjoribanks J, Lethaby A, Farquhar C (2006) Surgery versus medical therapy for heavy menstrual bleeding. Cochrane Database Syst Rev 2:CD003855

3. Abbott JA, Garry R (2002) The surgical management of menorrhagia. Hum Reprod Update 8:68-78

4. Rauramo I, Elo I, Istre O (2004) Long-term treatment of menorrhagia with levonorgestrel intrauterine system versus endometrial resection. Obstet Gynecol 104:1314-1321

5. Nagrani R, Bowen-Simpkins P, Barrington JW (2002) Can the levonorgestrel intrauterine system replace surgical treatment for the management of menorrhagia? BJOG 109:345-347

6. Hurskainen R, Teperi J, Rissanen P et al (2004) Clinical outcomes and costs with the levonorgestrel-releasing intrauterine system or hysterectomy for treatment of menorrhagia: randomized trial 5-year follow-up. JAMA 291:1456-1463

7. Tapper A-M, Heinonen PK (1995) Hysteroscopic endomyometrial resection for the treatment of menorrhagia-follow-up of 86 cases. Eur J Obstet Gynecol Reprod Biol 62:75-79
8. Cooper KG, Jack SA, Parkin DE, Grant AM (2001) Five-year follow up of women randomized to medical management or transcervical resection of the endometrium for heavy menstrual loss: clinical and quality of life outcomes. Br J Obstet Gynaecol 108:1222-1228

9. Boujida VH, Philipsen T, Pelle J, Joergensen JC (2002) Five-year follow-up of endometrial ablation: endometrial coagulation versus endometrial resection. Obstet Gynecol 99:988-992

10. Bøe Engelsen I, Woie K, Hordnes K (2006) Transcervical endometrial resection: long-term results of 390 procedures. Acta Obstet Gynecol Scand 85:82-87

11. Heinonen PK, Helin R, Nieminen K (2006) Long-term impact and risk factors for hysterectomy after hysteroscopic surgery for menorrhagia. Gynecol Surg 3:265-269

12. Lethaby A, Shepperd S, Cooke I, Farquhar C (1999) Endometrial resection and ablation versus hysterectomy for heavy menstrual bleeding. Cochrane Database Syst Rev 2:CD000329

13. Sowter MC, Singla AA, Lethaby A (2000) Pre-operative endometrial thinning agents before hysteroscopic surgery for heavy menstrual bleeding. Cochrane Database Syst Rev 2: CD001124

14. Roy KH, Mattox JH (2002) Advances in endometrial ablation. Obstet Gynecol Surv 57:789-802 